\title{
On the automorphism group of the countable dense circular order
}

by

\author{
J. K. Truss (Leeds)
}

\begin{abstract}
Let $(C, R)$ be the countable dense circular ordering, and $G$ its automorphism group. It is shown that certain properties of group elements are first order definable in $G$, and these results are used to reconstruct $C$ inside $G$, and to demonstrate that its outer automorphism group has order 2. Similar statements hold for the completion $\bar{C}$.
\end{abstract}

1. Introduction. Circular orders (or "cyclic orders") have been considered for instance in [1] and [2]. They have also arisen in the study of Jordan groups [4], and as examples of structures having rich automorphism groups [9]. Here I focus on a particular case, the countable dense circular order, written as $(C, R)$, which turns out to have particularly interesting properties, though some remarks apply to more general circular orders.

I begin by studying the group of all automorphisms of $(C, R)$. I am interested here in reproducing the results about reconstruction and outer automorphisms for the ordered rationals described for instance in [3].

As a first step, not strictly necessary for the development, though I hope of some interest, I give an analogue of Holland's description of the conjugacy classes for the ordered case in terms of "orbitals". Part of this is a reworking of classical material on orientation-preserving homeomorphisms of the unit circle in the complex plane $[5,8]$. In the next section I then give a list of first order formulae of the language of group theory which are devised with the intention of "reconstructing" the structure $(C, R)$ in its automorphism group. This leads in Section 4 to the results on reconstruction and outer automorphisms.

The definitions are as follows. By a circular order we understand a relational structure of the form $(C, R)$ where $R$ is a ternary relation on $C$ such

2000 Mathematics Subject Classification: Primary 06F99.

Key words and phrases: circular order, automorphism group. 
that $R(a, b, c)$ implies that $a, b$, and $c$ are distinct, and for each $a \in C$, the relation on the complement of $\{a\}$ given by $b<c \Leftrightarrow R(a, b, c)$ is a (strict) linear order, and $R(a, b, c) \Leftrightarrow R(b, c, a) \Leftrightarrow R(c, a, b)$. (Note that we allow the case where $C$ has fewer than three elements, when it is just the trivial structure on one or two points, since an "orbital pattern" - see below-may take one of these degenerate forms.) Throughout we shall assume that $C$ itself is countably infinite, and dense, which then determines it uniquely up to isomorphism. One may characterize "density" in this context either by saying that for any $a \neq b$ there must exist $c$ such that $R(a, c, b)$, or that it is obtained from the rationals by "bending round" the two ends to form a circle, formally $R(a, b, c)$ if $a<b<c \vee b<c<a \vee c<a<b$. It will be convenient at certain points to use other manifestations of $C$, for instance as the set of complex numbers of modulus 1 and rational argument (or even of integer argument, since this is clearly dense in the unit circle). We write $G$ for the automorphism group of $(C, R)$.

We may form the Dedekind completion $\bar{C}$ of $C$ by using the corresponding linear orders in the above definition. Thus if we pick $a \in C$, then $\{(b, c): R(a, b, c)\}$ is a linear order, which has a Dedekind completion as usual, and $\bar{C}$ is formed from this by replacing $a$, and saying that $R(x, y, z) \Leftrightarrow(x=a \wedge y<z) \vee(x<y<z)$ (or $x, y, z$ is a cyclic rearrangement of such a triple). In the countable dense case, $\bar{C}$ is then isomorphic to the unit circle in the complex plane under the natural circular ordering. Note that by density, any automorphism of $C$ induces an automorphism of $\bar{C}$, which we denote by the same letter.

Note that the automorphism group of the linear ordering $(\mathbb{Q},<)$ has two orbits in its action on $(\mathbb{R},<)$, namely the rationals and the irrationals. In a precisely analogous way, the automorphism group of $(C, R)$ has precisely two orbits in its action on $\bar{C}$, the points of $C$, and of $\bar{C}-C$, and we shall use this freely in what follows.

We shall also use interval notation, writing, for $a, b \in C,(a, b)=\{x \in C$ : $R(a, x, b)\}$ (or possibly the same set in $\bar{C}$ ), and similarly for closed or semiopen intervals. We visualize the relation $R$ as corresponding to the anticlockwise orientation, which fits with the usual orientation of the unit circle in the complex plane.

The referee has brought to my attention an unpublished paper [6] by McCleary and Rubin, in which the reconstruction parts of this paper are independently derived. The results in [6] are considerably more general than those presented here, not being confined to the particular case of the countable dense circular order. The methods given here could however be easily generalized, so this is not a major difference. I have concentrated exclusively on the countable case since the original intention (which may still 
be followed up) was to use the results to analyze the group of computable automorphisms, in the style of [7].

I now sum up the technical differences between the two papers. The representation used by McCleary and Rubin for elements of $\bar{C}$ is as the "initial points" of support sets of bounded elements. They do not (in the first instance, at any rate) restrict to elements having either just one or two fixed points as is done here (nor do they need to, nor can they, as that would mean that they could only represent points having countable coinitiality), nor to elements having just one bump. Finally, they show how to represent $\bar{C}$, but not $C$. Apart from these points, the conclusions in the case of a countable dense circular order are very similar, though the actual formulae used, and the routes to the conclusions adopted, are rather different.

2. Characterization of conjugacy. In this section we state and prove an analogue of Holland's "characterization of congugacy" (see [3, Theorem $2.2 .5]$ ). To state this correctly we require a few more definitions. There is a fundamental division of elements of $G=\operatorname{Aut}(C, R)$ into those having a fixed point in $\bar{C}$, and those not doing so. (We shall see in Section 3 that these two classes can be easily distinguished by a formula of the language of group theory.) If $g$ has a fixed point $x_{0}$, then it essentially acts as an element of the order-preserving automorphism group of its complement. In this case, we say that the orbital of an element $x$ is the set $\{y \in C$ : $\left.\exists m \exists n\left(y \in\left[g^{m} x, g^{n} x\right]\right)\right\}$, which is the convex set "spanned" by the orbit of $x$. (Sometimes this set will be evaluated in $\bar{C}$, and it is also sometimes referred to as an "interval of support".) The orbital has parity +1 if $R\left(x_{0}, x, g x\right)$, parity -1 if $R\left(x_{0}, g x, x\right)$, and parity 0 if $g x=x$. We also regard $\left\{x_{0}\right\}$ as an orbital of parity 0 . The family of all orbitals itself becomes a circular ordering on letting $R((x),(y),(z)) \Leftrightarrow R(x, y, z)$ (which one can check is independent of the choice of representatives), and the orbital pattern of $g$ is the circular ordering together with the colouring function which takes each orbital to its parity. Orbitals of parity 0 are called trivial. An element $g$ having just one non-trivial orbital is called a bump. This is a bounded bump if it also has a non-trivial interval of fixed points.

Now elements that have no fixed point in $\bar{C}$ are like "rotations" [8], though their rotational behaviour may be quite complicated to describe. In all cases it is shown in [8] how to associate with any $g \in G$ a "rotation number" $\alpha \in[0,1)$ which measures the "average" (anticlockwise) rotation of the action of $g$ on members of $\bar{C}$. Thus $g$ could be the rotation $\mathrm{e}^{\mathrm{i} \theta} \mapsto$ $\mathrm{e}^{\mathrm{i}(\theta+2 \pi \alpha)}$. The rotation number $\alpha$ is 0 if and only if $g$ has a fixed point in $\bar{C}$. Following on from this, $\alpha$ is rational if and only if $g$ has a finite cycle (orbit) in $\bar{C}$. In fact, if we write $\alpha=\frac{m}{n}$ in lowest terms, where $m$ and $n$ are positive 
integers, then $n$ is the least length of a finite cycle of $g$ in $\bar{C}$ (which includes as a special case $n=1$, in which case $m$ must be 0 , where $g$ has a fixed point, mentioned above). The members of the cycle $\left\{g^{i}(x): 0 \leq i<n\right\}$ are thus distinct points somehow arranged round $\bar{C}$, and a fundamental interval is any of the $n$ possible open intervals determined by these points. Note that the $n$ points of a cycle need not be arranged consecutively in the circular ordering (the orbit may "wind" round the origin more than once), but this information is provided by the angle of rotation, and in particular, by the value of $m$.

For elements $g$ such that no $g^{n}$ has any fixed points in $\bar{C}$, so that $\alpha$ is irrational, things are more complicated. We may regard the case in which all cycles are dense as the typical situation, but this does not necessarily happen. An analysis of the possible behaviour of orientation-preserving homeomorphisms of $\bar{C}$ was carried out by Poincaré, van Kampen, and others (see [8]), which mainly focussed on the case of no finite cycles. Since automorphisms of $(C, R)$ extend to $\bar{C}$, this classical theory applies here, though we have to modify it if we wish to give a complete description of such maps up to conjugacy in $\operatorname{Aut}(C, R)$, since the conjugacies may not preserve $C$ itself.

We briefly summarize the material from [8] and [5] that we need. The two main constructions of homeomorphisms of $\bar{C}$ to itself without finite cycles are these. We fix an irrational $\alpha \in(0,1)$. Then the rotation $g\left(\mathrm{e}^{\mathrm{i} \theta}\right)=\mathrm{e}^{\mathrm{i}(\theta+2 \pi \alpha)}$ is an obvious automorphism. We refer to an automorphism of $\bar{C}$ which is conjugate to this (or of $C$ which gives rise to such an element) as a pure rotation. Next let $X$ be any countable subset of $\bar{C}$ invariant under $g$ (a countable union of $g$-orbits). Then we may replace each point of $X$ by a copy of the closed interval $[0,1]$, and this becomes a circular order $\widetilde{C}$, isomorphic to $\bar{C}$ since it is complete with a countable dense subset. If $\varphi$ is the map from $\widetilde{C}$ to $\bar{C}$ which collapses each copy of $[0,1]$ to the point it replaced, then there is a natural homeomorphism of $\widetilde{C}$ which is obtained by lifting $g$ and mapping each point in a copy of $[0,1]$ to the corresponding point in its image. Automorphisms of $\bar{C}$ (or of $C$ ) arising in this way are called impure rotations. It is shown in $[8,5]$ that all homeomorphisms of $\bar{C}$ to itself without finite cycles are conjugate to a pure or impure rotation. Furthermore, in each case the angle $\alpha$ may be recovered from $g$, and for impure rotations the set $X$ may be too, up to rotational equivalence, by reversing the construction of $\widetilde{C}$ from $\bar{C}$ (see below). Thus two homeomorphisms without finite cycles are conjugate if and only if they are both pure or both impure, with the same angle of rotation, and in the second case if the sets $X$ are equivalent under some rotation (which is what is shown in [5]).

In our case we need to modify the above to obtain a complete characterization of conjugacy, since we are just considering actions on $C$ and not on $\bar{C}$. 
(In other words, there will be automorphisms of $(C, R)$ whose extensions to $\bar{C}$ are conjugate, but which are not conjugate in $\operatorname{Aut}(C, R)$ themselves.) For the case where there is a finite orbit, we have to distinguish the cases in which there is such an orbit in $C$, and where any such orbit is in $\bar{C}-C$. Apart from this, the finite cycle case is as for $\bar{C}$. For the other cases we need to define $\equiv$ on subsets of $\bar{C}$ or finite sequences of subsets of $\bar{C}$ by letting $X \equiv Y$ if there is some rotation (through any angle, rational or irrational) taking $X$ to $Y$.

First consider a pure rotation $g$ of $C$ through irrational $\alpha$ (we refer to it thus, though strictly speaking it is a rotation through $2 \pi \alpha$ ). Then by replacing it by a conjugate, $g$ becomes the map $g\left(\mathrm{e}^{\mathrm{i} \theta}\right)=\mathrm{e}^{\mathrm{i}(\theta+2 \pi \alpha)}$. Conjugation amounts to replacing $C$ by another countable dense subset $Y$ of $\bar{C}$, which one can show is independent of the choice of conjugacy, up to $\equiv$-equivalence, and is a countable union of orbits under rotation through $\alpha$. We say that such a set $Y$ is associated with $g$. Conversely, given any non-empty finite or countable union $Y$ of orbits of the action of rotation through $\alpha$ on $\bar{C}$, the restriction of $g$ to $Y$, which is isomorphic to $C$, is a pure rotation, which we denote by $g_{Y}$, and the set associated with $g_{Y}$ is $Y$.

For an impure rotation we may similarly consider any union of orbits of its action on $\bar{C}$. Since however we must use a dense subset of $\bar{C}$, and hence a dense subset of each copy of $[0,1]$, we may as well take just the rationals in each case. So what the choice depends on this time is the choice of a nonempty finite or countable union $X$ of orbits of $\bar{C}$ under rotation through $\alpha$ and another (this time possibly empty) finite or countable union $Y$ of such orbits disjoint from $X$. In addition we have to decide, for each orbit contained in $X$, whether to replace its points by (the rationals in) $I_{0}=[0,1], I_{1}=[0,1$ ), $I_{2}=(0,1]$, or $I_{3}=(0,1)$, so we need to specify a partitioning of $X$ into four unions of orbits $X_{0}, X_{1}, X_{2}$, and $X_{3}$ corresponding to these four cases. The quintuple $\left(X_{0}, X_{1}, X_{2}, X_{3}, Y\right)$ is associated with $g$. Once this choice has been made, we can determine an automorphism of $\bigcup_{i<4}\left(X_{i} \times I_{i}\right) \cup Y \cong C$ as before, denoted by $g_{X_{0}, X_{1}, X_{2}, X_{3}, Y}$. In the other direction, we need to see how $X_{0}, X_{1}, X_{2}, X_{3}, Y$ can be recovered from an impure rotation $g$. In [8] it is shown that in its action on $\bar{C}$, there is a unique minimal set $M$, being a non-empty union of orbits of $g$, which is the closure of each of these orbits (which, in the automorphism just described, consists of the union of the closure of $Y$ and the endpoints of intervals contributed by the members of $X$ ). In general, the minimal set for this impure rotation is homeomorphic to Cantor space, and if we identify endpoints of open intervals making up its complement, then we reach a pure rotation, through the same angle as $g$. Furthermore, we may "recover" $Y$ as the intersection with $C$ of the Cantor set that has been found, apart from any endpoints of intervals of its 
complement, and $X$ as the result of identifying the endpoints of all these intervals (in $\bar{C}$ ). The subdivision into $X_{0}, X_{1}, X_{2}$, and $X_{3}$ depends on which of these endpoints actually lie in $C$.

TheOrem 2.1. For any $f$ and $g$ in $G, f$ and $g$ are conjugate in $G$ if and only if one of the following holds:

(i) for some positive integer $n, f$ and $g$ each have a cycle of length $n$ of points of $C$, they have equal angles of rotation, and on some fundamental interval, the orbital patterns of $f^{n}$ and $g^{n}$ are isomorphic (this and the next clause include as a special case those automorphisms having a fixed point in $C$ or in $\bar{C}-C$, in which case the winding number is 0 , and the fundamental interval is the complement of the fixed point);

(ii) for some positive integer $n, f$ and $g$ each have a cycle of length $n$ of points of $\bar{C}-C$, but no finite cycles of points of $C$, they have equal angles of rotation, and on some fundamental interval, the orbital patterns of $f^{n}$ and $g^{n}$ are isomorphic;

(iii) $f$ and $g$ are both pure rotations through the same irrational angle $\alpha$, and their associated subsets of $\bar{C}$ are $\equiv$-equivalent;

(iv) $f$ and $g$ are both impure rotations through the same irrational angle $\alpha$, and their associated quintuples $\left(X_{0}, X_{1}, X_{2}, X_{3}, Y\right)$ are $\equiv-$ equivalent.

3. A list of formulae. In this section we give a list of first order formulae of the language of group theory, which are intended to express a variety of properties of members of $G=\operatorname{Aut}(C, R)$. We are aiming at interpreting $(C, R)$ inside its automorphism group (see the next section), which will be done without parameters up to orientation-reversal, and we represent a point of $C$ by any bounded bump of parity +1 having that point as initial point. With this in mind, the key things to express are " $x$ is a bounded bump" and "two bounded bumps have the same parity and initial point in the direction in which they act" (the formulae bump and sameinit respectively), and en route to this, formulae expressing that an automorphism has some fixed point, or exactly one or exactly two fixed points (in the completion) are also important. Other formulae are derived in a more standard way. A final key point is to distinguish the two orbits $C$ and $\bar{C}-C$, which is done using tricks similar to those for $\mathbb{Q}$.

There are clearly many more formulae that we could derive by similar methods (such as disjointness of arbitrary bumps) but we concentrate on what seems the quickest way to establish interpretability, though we do give a formula rot which suffices to characterize the pure rotations. Note that since our automorphisms act on the left, the notation for conjugates 
is $x^{y}=y x y^{-1}$.

- $\operatorname{fix}(x): \exists y\left(x^{2}=x^{y}\right)$ (in other words, $x$ is conjugate to its square),

- $\operatorname{rigid}(x, y): \operatorname{fix}(x) \wedge \operatorname{fix}(y) \wedge x y=y x \wedge \forall z \forall t(x z=z x \wedge x t=t x \wedge y z=$ $z y \wedge y t=t y \rightarrow z t=t z)$

- dense $(x):(\exists y) \operatorname{rigid}(x, y)$,

- onefix $(x): \exists y(\operatorname{rigid}(x, y) \wedge \forall z(x z=z x \wedge y z=z y \wedge z \neq 1 \rightarrow(\operatorname{rigid}(x, z) \vee$ $\operatorname{rigid}(y, z))))$,

- $\operatorname{twofix}(x): \neg \operatorname{onefix}(x) \wedge \exists y(\operatorname{rigid}(x, y) \wedge \forall z(x z=z x \wedge y z=z y \rightarrow$ $\left.\left.\left(\operatorname{rigid}(z, y) \vee \operatorname{rigid}(x z, y) \vee \operatorname{rigid}\left(x^{-1} z, y\right)\right)\right)\right)$,

- $\operatorname{bump}(x): x \neq 1 \wedge \neg \operatorname{dense}(x) \wedge \exists y \exists z(\operatorname{twofix}(y) \wedge \operatorname{rigid}(y, z) \wedge x y=$ $y x \wedge x z=z x)$,

- sameends $(x)$ : $\operatorname{bump}(x) \wedge(\exists y) \operatorname{twofix}\left(x x^{y}\right)$,

- $\operatorname{samepar}(x, y)$ : $\operatorname{sameends}(x) \wedge \exists z\left(y=x^{z}\right)$,

- $\operatorname{samesupp}(x, y): \operatorname{samepar}(x, y) \wedge \exists z(\operatorname{samepar}(x, z) \wedge \operatorname{twofix}(x z) \wedge$ twofix $(y z))$,

- $\operatorname{disj}(x, y)$ : sameends $(x) \wedge \operatorname{sameends}(y) \wedge \forall z(\operatorname{samesupp}(x, z) \rightarrow y z=z y))$,

- $\operatorname{subset}(x, y)$ : sameends $(x) \wedge \operatorname{sameends}(y) \wedge \operatorname{samepar}(x, y) \wedge \forall z(\operatorname{disj}(z, y)$ $\rightarrow \operatorname{disj}(z, x))$,

- initsubset $(x, y): \operatorname{subset}(x, y) \wedge \neg \exists z \exists t(\operatorname{samesupp}(y, z) \wedge \operatorname{disj}(x, t) \wedge$ $\left.\operatorname{subset}\left(t^{z}, x\right)\right)$,

- $\operatorname{sameinit}(x, y)$ : initsubset $(x, y) \vee \operatorname{initsubset}(y, x)$,

- $\operatorname{circ}(x, y, z): \exists x^{\prime} \exists y^{\prime} \exists z^{\prime}$ (sameinit $\left(x, x^{\prime}\right) \wedge \operatorname{sameinit}\left(y, y^{\prime}\right) \wedge \operatorname{sameinit}\left(z, z^{\prime}\right) \wedge$ $\operatorname{disj}\left(x^{\prime}, y^{\prime}\right) \wedge \operatorname{disj}\left(x^{\prime}, z^{\prime}\right) \wedge \operatorname{disj}\left(y^{\prime}, z^{\prime}\right) \wedge(\exists t)\left(\operatorname{samepar}(x, t) \wedge \operatorname{disj}\left(x^{\prime}, t\right) \wedge y^{t}\right.$ $=z)$,

- $\operatorname{rot}(x) ; \forall y \forall z(x y=y x \wedge x z=z x \rightarrow y z=z y)$.

Lemma 3.1. For any $f \in G, G \models \operatorname{fix}(f) \Leftrightarrow f$ has a fixed point in $\bar{C}$.

Proof. If $f$ has a fixed point in $\bar{C}$, then $f$ acts on the complement of the fixed point like an element of $\operatorname{Aut}(\mathbb{Q},<$ ), where it is well known (see [3] for instance) that every element is conjugate to its square.

Conversely, suppose that $f$ is conjugate to $f^{2}$, and let $\alpha \in[0,1)$ be its rotation number. Then it is clear that the rotation number of $f^{2}$ is $2 \alpha$ (where this is taken mod 1). But the rotation number is invariant under conjugacy, and hence $2 \alpha \equiv \alpha \bmod 1$. Since $0 \leq \alpha<1$ we deduce that $\alpha=0$, and hence $f$ has a fixed point in $\bar{C}$.

Lemma 3.2. For any $f, g \in G, G=\operatorname{rigid}(f, g) \Rightarrow f$ and $g$ each have at least one fixed point in $\bar{C}$, they have the same families of orbitals, and on each non-trivial orbital they act as translations by \pm 1 and $\alpha$ respectively, for some irrational number $\alpha$ (which may depend on the orbital).

Proof. Suppose that $G \models \operatorname{rigid}(f, g)$. Then by Lemma 3.1, $f$ and $g$ each have a fixed point in $\bar{C}$, so for each we may consider its family of orbitals. 
Since $f g=g f$, each of $f$ and $g$ preserves the family of orbitals of the other. Hence if $X$ and $Y$ are orbitals of $f$ and $g$ respectively that overlap, then one is a subset of the other. It follows that if $X \subset Y$ then $Y$ is a non-trivial orbital, and is a union of a set of orbitals of $f$, of which there must be at least two, and also that $g$ carries the action of $f$ on each such orbital to its action on the image. Consider two cases. In the first, all orbitals of $f$ inside $Y$ are trivial. Then the centralizer of $\langle f, g\rangle$ contains all elements of $G$ with support contained in $Y$ and commuting with $g$, and since this subgroup is isomorphic to $\operatorname{Aut}(\mathbb{Q},<)$ it is certainly not abelian. In the second case, there is a non-trivial such orbital $X$ say, and we can still embed $\operatorname{Aut}(\mathbb{Q},<)$ in the centralizer of $\langle f, g\rangle$ by letting it act on $(x, f(x))$ for some $x \in X$ (or $(f(x), x)$ if $f(x)<x)$ and extending to the whole of $X$ and then $Y$ by the commutation conditions, so the centralizer is still not abelian. In each case we contradict $\operatorname{rigid}(f, g)$. The conclusion is that $X=Y$. Similarly this follows if we assume that $X \supseteq Y$, and therefore $f$ and $g$ have the same orbitals.

Now consider the actions of $f$ and $g$ on one non-trivial orbital $X$. Assuming for ease that $f$ has parity +1 on $X$, we may view $\bar{X}$ as $\mathbb{R}$ and $f$ as translation by 1 . We see that $g$ may be taken to be a translation by an irrational number.

First suppose for a contradiction that some $\langle f, g\rangle$-orbit $Y$ of $X$ is not dense, and let $I$ be a maximal open interval of its complement. Consider the $\langle f, g\rangle$-orbit $\mathcal{I}=\left\{f^{m} g^{n} I: m, n \in \mathbb{Z}\right\}$ of $I$, all of whose elements are also maximal open intervals of the complement of $Y$. Let $J=\left\{(m, n) \in \mathbb{Z}^{2}\right.$ : $\left.f^{m} g^{n} I=I\right\}$. Since $f$ and $g$ commute, this is a subgroup of the group $\mathbb{Z}^{2}$, and since $I$ is not fixed by either $f$ or $g,(0,0)$ is the only member of $J$ having a zero co-ordinate. From this (by considering the least positive $m$ if any such that some $(m, n)$ is in $J)$ it readily follows that $J$ is cyclic, and hence consists of all multiples of some fixed $(m, n)$ (possibly $(0,0)$ ). Let $h_{1}$ and $h_{2}$ be non-commuting order-automorphisms of $I$ that each commute with $f^{m} g^{n}$ on $I$. Then by copying the action of $h_{i}$ on $I$ to each member $f^{r} g^{s} I$ of $\mathcal{I}$ using $f^{r} g^{s}$ and fixing all other points, we find still non-commuting order-automorphisms of $\mathbb{R}$ that both commute with $f$ and $g$, contrary to $G=\operatorname{rigid}(f, g)$. The conclusion is that every $\langle f, g\rangle$-orbit is dense.

To conclude this part of the argument, we appeal to some classical results as given in [8]. Since $f$ is translation by 1 , and $g$ commutes with it, they can be viewed as acting on the unit circle in $\mathbb{C}$ via the map $x \mapsto \mathrm{e}^{2 \pi \mathrm{i} x}$, and then $f$ is the identity. By the results given on pages 38-41 of [8] it follows that the action of $g$ on the circle is then conjugate to a rotation through $2 \pi \alpha$ for some irrational number $\alpha$. Lifting this back to $\mathbb{R}$, it follows that by replacing $g$ by a conjugate using a conjugacy that commutes with $f$, we may suppose that it is a translation by an irrational number, as desired. 
We remark that we cannot expect to prove the converse statement as it stands. Roughly speaking the state of affairs is this. Assume first that $f$ and $g$ have exactly the same orbitals, and that on each non-trivial orbital, if $f$ is viewed as translation by 1 then $g$ is translation by an irrational $\alpha$. If the irrationals arising for different non-trivial orbitals are all distinct, then certainly $G=\operatorname{rigid}(f, g)$ holds. It is clearly not necessary however that all the irrationals be distinct; for instance, there could be three non-trivial orbitals in all, two of them sharing the same irrational, distinct from that for the third, and then we would still have $G=\operatorname{rigid}(f, g)$. The precise statement would be rather complicated, and in any case we do not need it.

Lemma 3.3. For any $f \in G, G \models \operatorname{dense}(f) \Leftrightarrow f$ has at least one fixed point in $\bar{C}$ and its support is dense.

Proof. If $G \models$ dense $(f)$, let $g$ be such that $G \models \operatorname{rigid}(f, g)$. Then by Lemma 3.2, $f$ and $g$ have the same family of orbitals including the same fixed points. Suppose that $f$ does not have dense support. Then there is a non-trivial interval of points fixed by both $f$ and $g$. The centralizer of $\langle f, g\rangle$ contains all automorphisms of $C$ which fix all points outside this interval, and so it is non-abelian, contrary to $G \models \operatorname{rigid}(f, g)$.

Conversely, suppose that $f$ has a fixed point and dense support. It has at most $\aleph_{0}$ non-trivial orbitals, and we select distinct irrationals corresponding to these. We may now build $g$ having the same orbitals as $f$, and such that on each non-trivial orbital, if $f$ is viewed as translation by \pm 1 , then $g$ is translation by the irrational corresponding to that orbital. To see this, consider one of these orbitals $X$ with chosen irrational $\alpha$. Let $\left\{f^{n} x: n \in \mathbb{Z}\right\}$ be an orbit of $f$ and suppose for ease that $x<f x$. Then $(x, f x) \cong \mathbb{Q}+\mathbb{Q}$. $\alpha$, so inducing a corresponding identification of $\mathbb{Q}+\mathbb{Q} . \alpha$ with each $\left(f^{n} x, f^{n+1} x\right)$ by use of $f^{n}$ we obtain an identification of $\mathbb{Q}+\mathbb{Q} . \alpha$ with the whole of $X$ such that $f$ is translation by 1 , and then we can take the action of $g$ on $X$ to be translation by $\alpha$. To see that $G \models \operatorname{rigid}(f, g)$ and so dense $(f)$, note that $f$ and $g$ certainly commute since they have the same orbitals, and on each non-trivial orbital under the identification just made they both act as translations. If $h$ commutes with each, then $h$ must preserve the action of the pair $(f, g)$ on each orbital. By choice of the irrationals as all distinct, it follows that $h$ must actually fix each non-trivial orbital, and as the support is dense, also all trivial orbitals. Looking at $X$ which is identified with $\mathbb{Q}+\mathbb{Q} . \alpha$, we see by induction that for each $x \in X$ and integer $n, h(x+n)=h(x)+n$ and $h(x+n \alpha)=h(x)+n \alpha$, so $h(m+n \alpha)=h(0)+m+n \alpha$. Thus $h(x)-x$ is constant on a dense subset of $X$, so is constant throughout, from which it follows that $h$ is a translation. Therefore the centralizer of $\langle f, g\rangle$ is abelian. in $\bar{C}$.

Lemma 3.4. For any $f \in G, G=$ onefix $(f) \Leftrightarrow f$ has a unique fixed point $\bar{C}$. 
Proof. Suppose $G=\operatorname{onefix}(f)$ and let $g$ be such that $G=\operatorname{rigid}(f, g)$ and the other conditions apply. Then $f$ and $g$ have the same sets of orbitals. Suppose that they (each) have at least two non-trivial orbitals $X$ and $Y$. Then if $h$ is the restriction of $f$ to $X$, then $h \neq 1$ and $h$ commutes with both $f$ and $g$, but it does not have the same set of orbitals as either $f$ or $g$, so $G \models \neg \operatorname{rigid}(g, h)$ and $G \models \neg \operatorname{rigid}(f, h)$. We deduce that $f$ and $g$ each have just one non-trivial orbital. By Lemma 3.3 this is dense. In other words, $f$ and $g$ each have just one (the same) fixed point.

Conversely, suppose $f$ just fixes $x \in \bar{C}$, and let $X$ be the complement of $x$, so that $X$ is the unique non-trivial orbital of $f$, on which we may suppose it acts as translation by +1 . Let $g$ act as translation by an irrational $\alpha$. Then $G=\operatorname{rigid}(f, g)$, and if $h$ is a non-trivial member of $G$ commuting with both $f$ and $g$, then $h$ is also a translation, by $\beta$ say. Then $G=\operatorname{rigid}(f, h) \Leftrightarrow \beta$ is irrational, and $G \models \operatorname{rigid}(g, h) \Leftrightarrow \beta / \alpha$ is irrational. Since $\alpha$ is irrational, one of these must hold.

Lemma 3.5. For any $f \in G, G \models \operatorname{twofix}(f) \Leftrightarrow f$ has exactly two fixed points in $\bar{C}$.

Proof. Suppose that $G \models \operatorname{twofix}(f)$ and let $g$ be a witness for $y$. Then from the earlier work, and as $G \models \neg$ onefix $(f), f$ and $g$ have the same orbitals, and the union of their non-trivial orbitals, of which there are at least two, is dense. Suppose for a contradiction that there are three nontrivial orbitals $X, Y$, and $Z$, and that these are indexed by dense subsets of $\mathbb{R}$ in such a way that $f$ acts as translation by \pm 1 on each, and $g$ acts as translation by irrationals $\alpha, \beta$, and $\gamma$ respectively. Let $h$ act as $f$ on $X$, as $f^{-1}$ on $Y$, and fix all other points. Then $h$ commutes with each of $f$ and $g$, but $h, f h$, and $f^{-1} h$ each have a non-trivial interval of fixed points, so $G=\operatorname{rigid}(h, g) \vee \operatorname{rigid}(f h, g) \vee \operatorname{rigid}\left(f^{-1} h, g\right)$ is false. We deduce that $f$ has just two non-trivial orbitals whose union is dense, hence also exactly two fixed points (in $\bar{C}$ ).

Conversely, suppose that $f \in G$ has just two fixed points in $\bar{C}$, hence just two non-trivial orbitals $X, Y$ on which we may suppose that it acts as translation by \pm 1 (for ease we just treat the case of +1 ) and choose $g \in G$ having the same orbitals on which it acts as translation by distinct irrationals $\alpha, \beta$ respectively. Then if $h \in G$ commutes with both $f$ and $g$, it must fix each of $X$ and $Y$ and acts as a translation $h_{1}(t)=t+\gamma, h_{2}(t)=t+\delta$ on $X, Y$ respectively. We find that $G=\operatorname{rigid}(h, g) \Leftrightarrow \gamma / \alpha, \delta / \beta$ are both irrational, $G=\operatorname{rigid}(f h, g) \Leftrightarrow(1+\gamma) / \alpha,(1+\delta) / \beta$ are both irrational, and $G \models \operatorname{rigid}\left(f^{-1} h, g\right) \Leftrightarrow(-1+\gamma) / \alpha,(-1+\delta) / \beta$ are both irrational. If $G \models$ $\operatorname{rigid}(h, g) \vee \operatorname{rigid}(f h, g) \vee \operatorname{rigid}\left(f^{-1} h, g\right)$ is false then, from $G=\neg \operatorname{rigid}(h, g)$, $\gamma / \alpha$ or $\delta / \beta$ is rational. Suppose the former. Then $(1+\gamma) / \alpha$ is irrational, so from $G \models \neg \operatorname{rigid}(f h, g)$ we deduce that $(1+\delta) / \beta$ is rational, but then 
$(-1+\gamma) / \alpha$ and $(-1+\delta) / \beta$ are both irrational, so $G \models \operatorname{rigid}\left(f^{-1} h, g\right)$ after all. The conclusion is that $G \models \operatorname{twofix}(f)$ as desired.

Lemma 3.6. For any $f \in G, G \models \operatorname{bump}(f) \Leftrightarrow f$ is a bounded bump.

Proof. First suppose that $G \models \operatorname{bump}(f)$, and let $g$ and $h$ be witnesses for $y$ and $z$. Then by Lemma $3.5, g$ has just two fixed points in $\bar{C}, a$ and $b$ say, and by Lemma 3.2 , on $(a, b)$ and $(b, a), g$ and $h$ may be viewed as acting as translations by \pm 1 and $\alpha, \beta$ respectively, where $\alpha$ and $\beta$ are irrationals. As in the proof of Lemma 3.2, $f$ must also act as a translation on each of $(a, b)$ and $(b, a)$. Since $G=f \neq 1 \wedge \neg$ dense $(f)$, one of these translations must be trivial, and the other non-trivial, and so $f$ is a bounded bump.

Conversely, suppose that $f$ is a bounded bump, with support $(a, b)$. Then we can build $g, h \in G$ such that $f$ and $g$ agree on $(a, b)$, and such that $g, h$ act as translations by \pm 1 and $\alpha, \beta$ respectively on $(a, b)$ and $(b, a)$, where $\alpha$ and $\beta$ are distinct irrationals. Then $g$ and $h$ are witnesses for $y$ and $z$ in the formula, and hence $G \models \operatorname{bump}(f)$ as required.

Lemma 3.7. For any $f \in G, G=\operatorname{sameends}(f) \Leftrightarrow f$ is a bounded bump the endpoints of whose support either both lie in $C$, or both lie in $\bar{C}-C$ (we say that it has the "same ends").

Proof. Suppose that $f$ is a bounded bump having the same ends and support $(a, b)$. Then there is an automorphism $g$ interchanging $a$ and $b$, and clearly $G \mid=\operatorname{twofix}\left(f f^{g}\right)$.

Conversely, if $G \models$ sameends $(f)$, let $g$ be a witness for $y$. Then $f$ is a bounded bump with support $(a, b)$ say, and so $f^{g}$ is a bounded bump of the same parity as $f$ and with support $(g a, g b)$. Since $G \models \operatorname{twofix}\left(f f^{g}\right)$, $(a, b) \cup(g a, g b)$ is dense. Hence $(b, a) \cap(g b, g a)=\emptyset$. Therefore $f f^{g}$ agrees with $f$ on $(g b, g a)$ and with $f^{g}$ on $(b, a)$, so has no fixed points in either of these sets. Furthermore, as $f$ and $f^{g}$ have equal parity, $f f^{g}$ has no fixed points in $(g a, b)$ or $(a, g b)$ either. So the only possible fixed points are $a, b, g a, g b$. But if $g a \neq b$ then none of these is fixed, since then $f$ moves $g a$ and $f^{g}$ does not, and $f^{g}$ moves $b$ and $f$ does not, and similarly for $a$ and $g b$. Since $f f^{g}$ has exactly two fixed points, it follows that $g a=b$ and $a=g b$. We deduce that $a$ and $b$ are in the same orbit of $G$, so either they are both in $C$ or both in $\bar{C}-C$.

Let us say that two bounded bumps are equivalent if they have the same parity, and either all four of their ends lie in $C$, or they all four lie in $\bar{C}-C$. This notion of equivalence is thus only defined on certain bumps, but these are the ones we want to use for the interpretation, so this suffices.

Lemma 3.8. For any $f, g \in G, G \models \operatorname{samepar}(f, g) \Leftrightarrow f$ and $g$ are equivalent bounded bumps. 
Proof. This is just the same as sameends for $f$ except that any conjugate of such a bounded bump must have its ends in the same one of $C$ or $\bar{C}-C$ as $f$, since members of the automorphism group of $C$ preserve each of $C$ and $\bar{C}-C$.

Lemma 3.9. For any $f, g \in G, G \models \operatorname{samesupp}(f, g) \Leftrightarrow f$ and $g$ are equivalent bounded bumps of equal support.

Proof. Suppose $f$ and $g$ are equivalent bounded bumps of equal support $(a, b)$, where $a$ and $b$ both lie in $C$, or both lie in $\bar{C}-C$. Let $h$ be a bump also of the same parity having support $(b, a)$. Then $f, g$, and $h$ are all conjugate, and $G=\operatorname{twofix}(f h) \wedge \operatorname{twofix}(g h)$.

Conversely, suppose that $G \models$ samesupp $(f, g)$, and let $h$ be a witness for $z$. Then $f, g$ and $f^{h}$ are bounded bumps of equal parity, and if their supports are $\left(a_{1}, b_{1}\right),\left(a_{2}, b_{2}\right)$, and $(c, d)$ respectively, then $a_{1}, b_{1}, a_{2}, b_{2}, c, d$ either all lie in $C$, or all lie in $\bar{C}-C$. Since $G=\operatorname{twofix}\left(f f^{h}\right)$, we can show by the same argument as used in Lemma 3.7 that $c=b_{1}$ and $a_{1}=d$, and it similarly follows that $c=b_{2}$ and $a_{2}=d$, and we deduce that $a_{1}=a_{2}$ and $b_{1}=b_{2}$, so that $f$ and $g$ have equal supports.

Lemma 3.10. For any $f, g \in G, G=\operatorname{disj}(f, g) \Leftrightarrow f$ and $g$ are bounded bumps having disjoint supports and the same ends.

Proof. In one direction this follows from the fact that permutations with disjoint supports commute. Conversely, suppose that $G \models \operatorname{disj}(f, g)$. Then $f$ and $g$ are indeed bounded bumps each having the same ends. If their supports are not disjoint then we can easily find an automorphism having the same support as $f$ which does not commute with $g$.

Lemma 3.11. For any $f, g \in G, G \models \operatorname{subset}(f, g) \Leftrightarrow f, g$ are equivalent bounded bumps, and $\operatorname{supp} f \subseteq \operatorname{supp} g$.

Proof. The only point to notice here is that if the support of $f$ is not a subset of the support of $g$, then there is a bounded bump having the same ends whose support is disjoint from that of $g$ but not of $f$.

Lemma 3.12. For any $f, g \in G, G \models \operatorname{initsubset}(f, g) \Leftrightarrow f, g$ are equivalent bounded bumps having the same initial points of support (taking "initial" to mean in the orientation given by their common parity) with supp $f \subseteq$ $\operatorname{supp} g$.

Proof. Suppose that $f$ and $g$ are equivalent bounded bumps with supp $f$ $\subseteq \operatorname{supp} g$, but with different initial points of support, $a$ and $b$ say. Then there is another equivalent bounded bump $k$ with support contained in $(b, a)$, and a bounded bump with the same support as $g$ which conjugates $k$ to an element whose support is contained in that of $f$. Thus initsubset $(f, g)$ is 
false. Conversely, if such a $k$ exists, then we see that $f$ and $g$ cannot have the same initial point of support.

Lemma 3.13. For any $f, g \in G, G \models \operatorname{sameinit}(f, g) \Leftrightarrow f, g$ are bounded bumps of equal parities having the same initial point of supports.

Lemma 3.14. For any $f, g, h \in G, G=\operatorname{circ}(f, g, h) \Leftrightarrow f, g, h$ are equivalent bounded bumps of equal parities such that either this parity is +1 and $R(a, b, c)$, or the parity is -1 and $R(a, c, b)$, where $a, b, c$ are the initial points of the supports of $f, g, h$.

Lemma 3.15. For any $g \in G, G=\operatorname{rot}(g) \Leftrightarrow$ there is an isomorphism $\theta$ from $(C, R)$ to some countable dense cyclic order $\left(C^{\prime}, R^{\prime}\right)$ so that $\theta g \theta^{-1}$ is a rotation through an irrational multiple of $\pi$.

Proof. Suppose that $f$ acts on $\bar{C}$ as a rotation through $2 \pi \alpha, \alpha$ irrational. Then $X=\left\{f^{n} 0: n \in \mathbb{Z}\right\}$ is dense (since if not, there is a non-empty maximal open interval $I$ of its complement with positive measure $\varepsilon$, and as $C$ has finite measure there is $n \neq 0$ such that $f^{n} I \cap I \neq \emptyset$, which by maximality gives $f^{n} I=I$, contrary to $\alpha$ being irrational). Suppose that $g$ commutes with $f$, and let $g(0)=\mathrm{e}^{\mathrm{i} \beta}, h\left(\mathrm{e}^{\mathrm{i} \theta}\right)=\mathrm{e}^{\mathrm{i}(\theta+\beta)}$ for all $\theta$. Then $g\left(f^{n} 0\right)=f^{n} g(0)=f^{n} \mathrm{e}^{\mathrm{i} \beta}=\mathrm{e}^{\mathrm{i}(\beta+2 \pi n \alpha)}=h\left(f^{n} 0\right)$. Hence $g$ and $h$ agree on a dense set, so are equal. Therefore any element commuting with $f$ is a rotation, and hence its centralizer is abelian, establishing $G=\operatorname{rot}(f)$.

Conversely, suppose that $G=\operatorname{rot}(f)$, and consider the action of $f$ on $\bar{C}$. We appeal to Theorem 2.1. We see that $f$ cannot have any finite cycle, as then its centralizer would not be abelian, and the same applies to the case given in 2.1(iv). We deduce that case (iii) of the theorem must apply, so $f$ is conjugate to a rotation through an irrational multiple of $\pi$, as desired.

4. Interpreting $(C, R)$ in its automorphism group. In this section we show how to use the formulae whose properties were developed in the previous section to provide an interpretation of $(C, R)$ in $G$ (up to reversal of the orientation), and from this we deduce that its outer automorphism group has order 2 . To complete the interpretation, we require two more formulae:

- $\operatorname{grid}(x, y): \operatorname{rigid}(x, y) \wedge$ onefix $(x)$,

- countorbit $(x)$ : $\operatorname{bump}(x) \wedge \exists y \exists z\left(\operatorname{grid}(y, z) \wedge \forall t \exists u\left(y u=u y \wedge z u=u z \wedge \operatorname{sameinit}\left(x^{t}, x^{u}\right)\right)\right)$.

Lemma 4.1. For any $f, g \in G, G \models \operatorname{grid}(f, g) \Leftrightarrow f$ and $g$ both have the same unique fixed point in $\bar{C}$, and on its complement they act as translations through 1 and some irrational (under a suitable identification of this complement with $\mathbb{R}$ ). 
Proof. First suppose that $G \models \operatorname{grid}(f, g)$. Since $G \models \operatorname{rigid}(f, g), f$ and $g$ have the same support, and as $G \models$ onefix $(f), f$ has just one fixed point in $\bar{C}$, which is therefore also the unique fixed point of $g$. The rest follows by Lemma 3.2.

Conversely, if $f$ and $g$ have the stated form, then their joint centralizer consists just of translations (under the identification made with $\mathbb{R}$ ), and so the formula grid is satisfied.

Lemma 4.2. For any $g \in G, G=$ countorbit $(f) \Leftrightarrow f$ is a bump with the initial point of its support lying in $C$.

Proof. Suppose that $G=$ countorbit $(f)$, and let $g$ and $h$ be witnesses for $y$ and $z$, which therefore satisfy grid. Let $a$ be the unique fixed point of $g$ and $h$, and identify $\bar{C}-\{a\}$ with $\mathbb{R}$ so that $g$ and $h$ are translations through 1 and an irrational $\alpha$ so that the points of $\mathbb{R}$ corresponding to points of $C$ are precisely of the form $m+n \alpha$ for integers $m$ and $n$. Then the centralizer of $\langle g, h\rangle$ consists of all translations which fix $C$ setwise. This centralizer is therefore countable. The condition in countorbit implies that the orbit of the initial point of the support of $f$ is countable and hence it lies in $C$.

Conversely, suppose $f$ is a bump the initial point $b$ of whose support lies in $C$, and choose $g, h$ such that $\operatorname{grid}(g, h)$ and the fixed point $a$ of $g, h$ is different from $b$. In addition suppose that $g, h$ are chosen so that $C$ is an orbit of $\langle g, h\rangle$. Then these are witnesses as required to verify countorbit since any conjugate of $f$ by a member of $G$ takes $b$ to a member $c$ of $C$, so there is a member of $\langle g, h\rangle$ taking $b$ to $c$, witnessing $u$.

TheOREM 4.3. There are interpretations of $C$ in $\operatorname{Aut}(C, R, g)$ and of $\bar{C}$ in $\operatorname{Aut}(\bar{C}, R, f)$ for suitable $f \in \operatorname{Aut}(C, R)$ and $g \in \operatorname{Aut}(\bar{C}, R)$.

Proof. We represent the members of $C$ by either of the two conjugacy classes of bounded bumps satisfying countorbit, where these are identified if they have the same initial point, and under the relation circ. These two conjugacy classes correspond to the two possible orientations of the circle. Since we are allowed to choose a parameter, we can choose one which distinguishes between the two orientations, specifically we let $f$ be one element satisfying countorbit with positive orientation (i.e. it acts in an anticlockwise direction), and then we can ensure that the conjugacy class used is the one containing $f$.

The same methods apply to $\bar{C}$, except that it is a little simpler, since there is only one orbit of initial points of elements satisfying bump, so we can just use elements satisfying bump, identified if they have the same initial point, and under the relation circ.

TheOREM 4.4. Suppose that $\left(C_{1}, R_{1}\right)$ and $\left(C_{2}, R_{2}\right)$ are countable dense circular orders, and $\theta$ is an isomorphism from $\operatorname{Aut}\left(C_{1}, R_{1}\right)$ to $\operatorname{Aut}\left(C_{2}, R_{2}\right)$. 
Then there is a bijection $f$ from $C_{1}$ to $C_{2}$ which is either orientationpreserving or orientation-reversing, and such that $\theta(g)=f g f^{-1}$ for every $g \in \operatorname{Aut}\left(C_{1}, R_{1}\right)$. A similar statement holds for $\bar{C}_{1}$ and $\bar{C}_{2}$. Hence the outer automorphism groups of $\operatorname{Aut}(C, R)$ and $\operatorname{Aut}(\bar{C}, R)$ have order 2.

Proof. Looking at the formulae we have constructed, we see that the image under $\theta$ of any bounded bump must be a bounded bump. If $\theta$ reverses the parity of some (and hence, as is easy to see, every) bounded bump, we compose with a single orientation-reversing automorphism to reduce to the case where it preserves the parity of every bounded bump. The remaining formulae considered show that $\theta$ determines an automorphism of $(C, R)$, and standard arguments (for instance see [10]) show that $\theta$ is actually conjugation by this automorphism.

Acknowledgements. I am grateful to Andrei Morozov for fruitful discussions about this paper during his visit to Leeds, supported by a Scheme 2 grant from the London Mathematical Society.

\section{References}

[1] M. Droste, M. Giraudet, and D. Macpherson, Periodic ordered permutation groups and cyclic orderings, J. Combin. Theory Ser. B 63 (1995), 310-321.

[2] M. Giraudet and W. C. Holland, Ohkuma structures, Order 19 (2002), 223-237.

[3] A. M. W. Glass, Ordered Permutation Groups, London Math. Soc. Lecture Note Ser. 55, Cambridge Univ. Press, 1981.

[4] D. Macpherson, A survey of Jordan groups, in: Automorphisms of First-Order Structures, R. Kaye and D. Macpherson (eds.), Oxford Univ. Press, 1994, 73-110.

[5] N. G. Markley, Homeomorphisms of the circle without periodic points, Proc. London Math. Soc. 20 (1971), 688-698.

[6] S. McCleary and M. Rubin, Locally moving groups and the reconstruction problem for chains and circles, arXiv:math/0510122.

[7] A. S. Morozov and J. K. Truss, On computable automorphisms of the rational numbers, J. Symbolic Logic 66 (2001), 1458-1470.

[8] Z. Nitecki, Differentiable Dynamics, MIT Press, 1971.

[9] J. K. Truss, Generic automorphisms of homogeneous structures, Proc. London Math. Soc. 64 (1992), 121-141.

[10] - On recovering structures from quotients of their automorphism groups, in: Ordered Groups and Infinite Permutation Groups, W. C. Holland (ed.), Kluwer, 1996, 63-95.

Department of Pure Mathematics

University of Leeds

Leeds, UK

E-mail: J.K.Truss@leeds.ac.uk

Received 24 July 2007;

in revised form 17 June 2008 and 12 March 2009 